\title{
Low-power computation methods and self-powered systems for Structural Health Monitoring techniques using Lamb waves for embedded sensing
}

\author{
M. Lallart ${ }^{\mathrm{a} *}$, D. Guyomar ${ }^{\mathrm{a}}$ and T. Monnier ${ }^{\mathrm{b}}$ \\ ${ }^{a} L G E F$, INSA-Lyon, Villeurbanne, France \\ ${ }^{\mathrm{b}}$ MATEIS, INSA-Lyon, Villeurbanne, France
}

\begin{abstract}
Recent trends in Structural Health Monitoring (SHM) systems have demonstrated the growing interest in autonomous, embeddable devices able to perform in-situ inspection and to work conjointly and wirelessly in a global sensor network. Hence, the energy aspect is of prior importance when dealing with such systems. However, most signal processing methods for assessing the integrity of a structure still rely on linear transforms or operators, such as Fourier or amplitude analysis, which for example require analog-to-digital converters that consume a lot of energy. As well, in addition of optimizing the signal processing, another important part is the use of a proper energy management for the whole system. Hence, the purpose of this paper is twofold. First, it aims at reviewing a particular set of low energy cost methods for Structural Health Monitoring consisting of deriving a Damage Index (DI) obtained from the comparison of the LW signature with a reference signal using logical operators that greatly reduce the required computational energy. A particular emphasis will be placed not only on the performance of the exposed techniques, but also on their energy consumption as well as their sensitivity and robustness facing environmental drifts. It will therefore be shown that the use of non-linear operators for the comparison permits ultra-low energy requirements while allowing detection abilities similar to classical approaches. The second objective of this paper is to discuss the implementation of the whole system in an efficient way using proper management strategies. This issue will be discussed through an example of a self-powered SHM scheme powered using ambient vibrations.
\end{abstract}

Keywords: Structural Health Monitoring, damage index, Lamb wave, low-power, self-powered

\section{Introduction}

The increasing demand in safety and reliability in transportations and civil engineering have required a redefinition of Structural Health Monitoring (SHM) standards. Coupled with the constant decrease in energy requirements of electronic devices, this has permitted the conception of in-situ monitoring systems, able to perform inspection in real time and in an autonomous fashion. However, powering such systems still remains an open issue. In particular, the use of primary batteries is problematic as such a solution requires regular maintenance [1] and raises environmental problems because of their complex recycling process.

In order to tackle the drawbacks of batteries, harvesting energy from the close environment has been proposed. Among the available sources, a particular attention has been placed on vibration energy scavenging using piezoelectric materials, as vibrations are widely available and as piezoelectric transducers features relatively high energy

\footnotetext{
*Corresponding author: M. Lallart, LGEF, INSA-Lyon, 8, rue de la Physique, F-69621, Villeurbanne, France. E-mail: mickael.lallart@ insa-lyon.fr.
} 
densities and promising integration potentials [2]. Nevertheless, the energy that can be harvested is limited in the range of tens of microwatts to a few milliwatts.

Therefore, to dispose of self-powered SHM systems, the energy balance between harvesting abilities and required energy for the inspection should be positive. Unfortunately, most of the in-situ monitoring approaches developed so far (for instance Lamb waves, impedance methods, acoustic emission, amplitude analysis [3-6]) requires much more energy than that can be delivered by the microgenerator, as they necessitate complex algorithms.

In addition, it can be noted that in real working conditions, environmental factors such as pressure or temperature would also modify the signature of the acoustic wave, as the DI approach relates the evolution of the structural state and not exactly the presence of a damage. However, changes caused by such parameters are slow and the evolution of the DI would be smooth, while the occurrence of a damage would abruptly modify the DI. Hence, by tracking the change of the DI in a relatively limited time period, it is possible to separate environmental factors that lead to small changes, with the occurrence of a damage which causes abrupt changes. This also shows the need of very low-power systems, as the control should be done relatively often to separate the two processes, and therefore enough energy should be harvested in a minimum of time.

Consequently, it is necessary to re-think SHM schemes to meet the power balance and therefore to be able to perform Structural Health Monitoring using a very few amount of energy. Hence, the purpose of this paper is first to expose low-cost but efficient SHM techniques based on simple computations of the Lamb wave signature received on a sensor. It will be shown that nonlinear processing using logical operators permits a dramatic reduction of the energy consumption of the inspection method while preserving the sensitivity of the detection.

In addition to the optimization of the DI computation, the power management strategy controlling the global operations of the system is also a significant issue for reducing the energy cost of the system. The second part of this paper (Section 5) therefore aims at exposing through a truly working self-powered system design rules for limiting the energy requirements of the global system, showing that the ability of performing self-powered wireless SHM is no longer chimerical.

\section{Principles and general formulation of the Damage Index}

The aim of this section is to provide the basics of the inspection scheme that will be described in this paper. Then a generalized approach will be developed in a mathematical manner, in order to easily identify each operator and therefore to optimize the computation requirements.

\subsection{Monitoring method}

The principle of the considered monitoring scheme relies on the interaction of an acoustic wave (Lamb wave) with the host structure (Fig. 1). The Lamb wave is typically generated by applying a voltage on a piezoelectric element acting as an emitter and sensed using a second piezoelectric material. As the wave travels through the structure, its interaction with the material would be different whether the structure is healthy or damaged and therefore the sensed signature would change as well. This interaction also changes as the damage growths. Hence, from the comparison of the sensed signature with a reference, it is possible to assess the condition of the host structure.

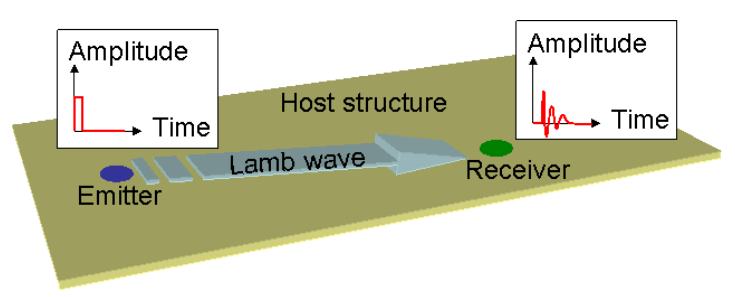

Fig. 1. Principles of the Lamb wave-based inspection. 
Therefore such an approach permits the computation of the relative condition of the structure, giving the evolution of the structural state rather than giving the absolute condition. In addition, this method requires the active driving of the piezoelectric element, therefore giving a snapshot of the structural state at a given moment. This event-driven approach is therefore suitable for low-power SHM. The energy required to generate the Lamb wave is moderate as well (usually much less than $1 \mathrm{~mJ}$ ), and can range up to a few meters.

\subsection{Generalized Damage Index}

The principles of the monitoring scheme consists of comparing the received signature of the acoustic wave on the sensor with a reference signature, leading to the definition of a Damage Index $(D I)$ that consists of applying a distance operator $g$ on the transformed signature and reference signals [7]:

$$
D I=g(f(\operatorname{sig}), f(r e f))
$$

where sig and ref refer to the sensed and reference signals, and $f$ to the transform operator. The Damage Index is usually bounded between 0 (pristine case) and 1 (fully broken structure). The distance operator can be defined in several ways, such as absolute difference, squared difference, absolute difference of the squared signals. If the signal transform is a vector, an integration (or Sun) may also be used in the distance operator. Usually, the transform consisted in applying the discrete Fourier transform of the signals [6]. However, such an approach requires lot of computations, as the number of operations for the FFT is $\mathrm{O}\left(N \cdot \log _{2}(N)\right)$ operations, with $\mathrm{N}$ the number of samples [8], therefore making it inefficient in terms of power savings. Another method would consist of using discrete wavelet transform which requires $\mathrm{O}\left(\operatorname{Nog}_{2}(L)\right)$ operations (with $L$ the scale depth or filter length) or RMS value $(\mathrm{O}(N)$ operations). Hence, the mathematical, high-level formulation of Eq. (1) shows that the transform operator is the most energy-consuming part. In order to further reduce the cost of this operator which requires more computation than the distance operator, it will be shown that nonlinear approaches may be highly beneficial.

\section{Low-power Damage Index through nonlinear processing}

The purpose of this section is to present nonlinear transform operators for the computation of the Damage Index. The proposed methods will rely on Boolean operations based on simple comparisons of the signal. Two approaches will be exposed: either the transform will consist in comparing the absolute value of the signature with a given threshold, or the zero-crossing time instants will be compared with a reference zero-crossing time instant vector.

\subsection{DI based on time above a threshold}

The first nonlinear method that will be considered in this study consists in comparing the absolute value of the sensed signature with a preselected threshold value [9] (Fig. 2a) using a logical operation based on "greater or equal" comparison. The comparison with the threshold therefore gives a signal whose value is either 0 or 1 , whose value is then summed and compared with the reference value:

$$
D I=\frac{\sum_{k}\left(\mid \text { ref }_{k} \mid>\text { thres }\right)-\sum_{k}\left(\mid \text { sig }_{k} \mid>\text { thres }\right)}{\sum_{k}\left(\left|r e f_{k}\right|>\text { thres }\right)}
$$

where thres refers to the threshold value, whose value is usually comprised between a tenth to nine tenth of the maximal value of the reference signature. 


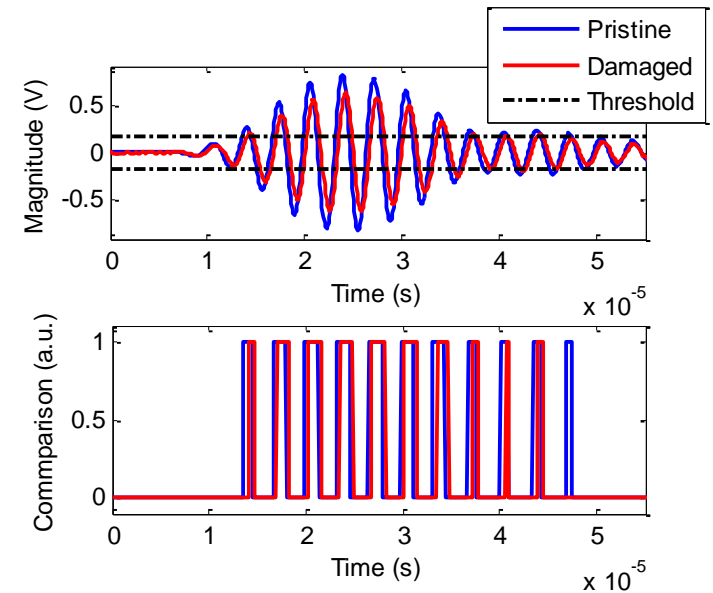

(a)
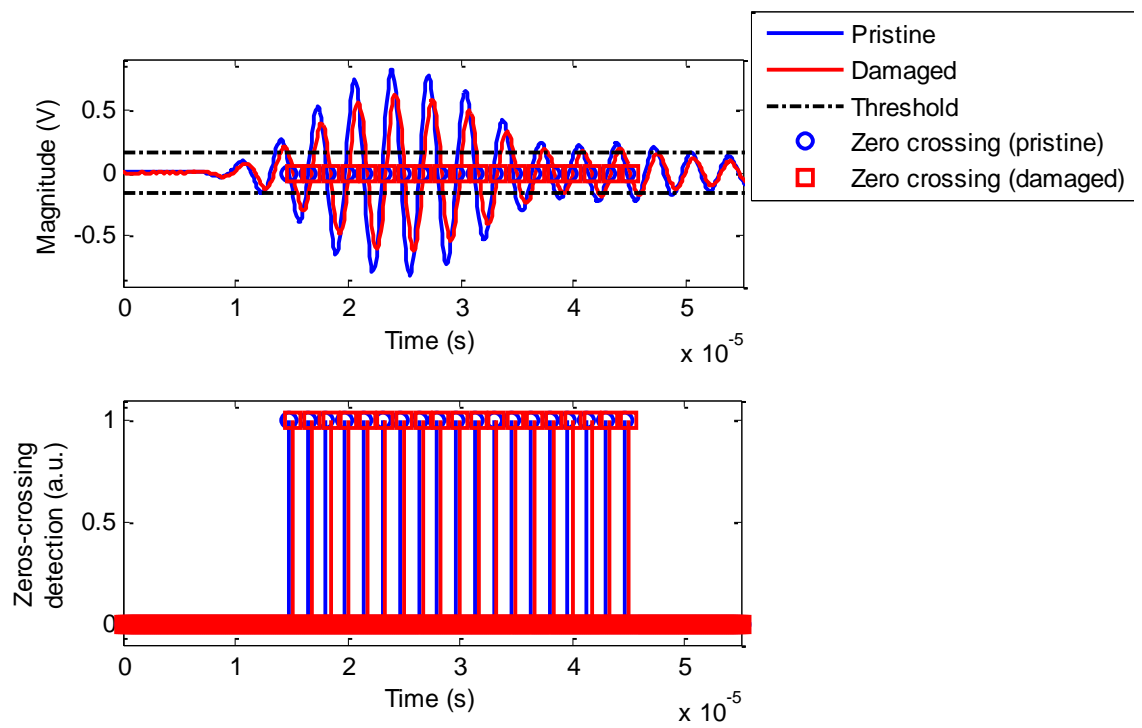

(b)

Fig. 2. Principles of the nonlinear DIs: (a) threshold-based; (b) zeros-crossing time instant-based.

\subsection{DI based on zero crossing time instants}

Another approach for lowering the power consumption of the computation of the Damage Index lies in comparing the zero-crossing time instants [10], as zero-crossing detection is simple enough to be implemented in a very-low power fashion. In addition, to ensure the robustness of the system facing noise, the zero crossing detection is only enabled when the voltage reaches a predefined threshold value (Fig. 2b).

The definition of the DI in this case thus yields:

$$
D I=\frac{\sum_{k}\left(t_{r e f_{k}}-t_{s i g_{k}}\right)}{\sum_{k} t_{r e f_{k}}}
$$

with $t_{r e f}$ and $t_{s i g}$ the reference and sensed signature zero-crossing time instant vectors. 


\section{Performance comparison}

The purpose of this section is to compare the operations of the proposed Damage Indexes with classical implementations of the estimator using FFT or RMS. The performance evaluation has been done using a CRFP composite plate equipped with two piezoelectric elements acting as emitter and three acting as receivers (Fig. 3). According to the used emitter/receiver pair, the damage location with respect to the Lamb wave path can be selected between on path/on fiber axis, on path/off fiber axis and off path/off fiber axis. A damage consisting of a fully penetrating hole with a growing diameter has then been inserted in the structure. 100 measurements have been done for the pristine case, and then 10 for each damage diameter. Three different signals have been used for the excitation of the emitter: sine burst with five cycles at $300 \mathrm{kHz}$ which corresponds to the first symmetrical mode $S_{0}$, sine burst with five cycles at $80 \mathrm{kHz}$ which corresponds to the first anti-symmetrical mode $A_{0}$, and pulse excitation.
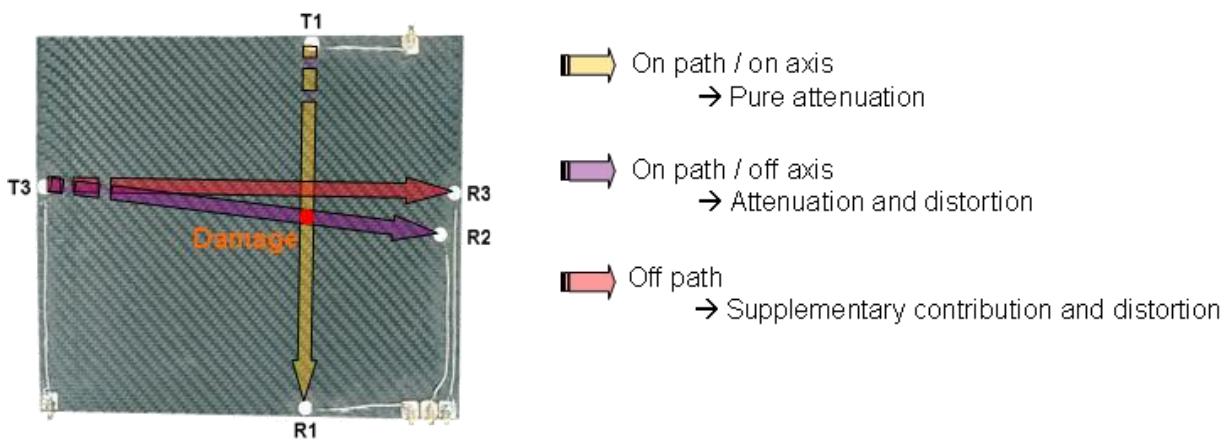

Fig. 3. Experimental structures for DI comparison.

\subsection{Evolution of the DIs as a function of the damage}

Figure 4 depicts the evolution of the four considered Damage Indexes (FFT-based, RMS-based, threshold comparison and zero-crossing time instant) for each possible configuration allowed by the experimental test structure. These charts clearly demonstrate that the DI based on the time period when the signal is above a threshold performs almost exactly as the classical DI implementation, and therefore allows determining the evolution of the structural condition. In particular, it adopts the same trend of having an important variation for low damages, which therefore permits early detection. Then, as the damage grows to higher importance, the classical DIs and nonlinear approach based on threshold detection tend to saturate.

Contrary to the three previous case, the zero-crossing time instant detection Damage Index tends to have a linear evolution as a function of the damage diameter in most of the cases, which permits a better appreciation of the damage severity. Although the value of this DI is less than the other ones, its standard deviation is limited and therefore allows a good monitoring.

Therefore, the nonlinear DIs permits detecting damage with similar performance compared to classical implementations of the method which require costly computation algorithms. The damage detection is performing well whatever the position of the damage with respect to the emitter/transmitter pair, even if the defect is not on the direct path between the emitter and the receiver, as the default introduces supplementary reflections that are sensed by the piezoelectric element used as receiver.

Finally, it can be noticed that the wavelength of the Lamb waves, equal to $2.3 \mathrm{~cm}$ for the $A_{0}$ mode and $1.9 \mathrm{~cm}$ for the $S_{0}$ mode, are largest than the larger damage size, which therefore indicates that the DI-based monitoring method allows detecting defects smaller than the wavelength.

\subsection{Implementation issues}

In addition to the performance in detecting the evolution of the structural changes exposed in the previous section, implementation issues of the proposed approaches are also discussed. In particular, with their application to 

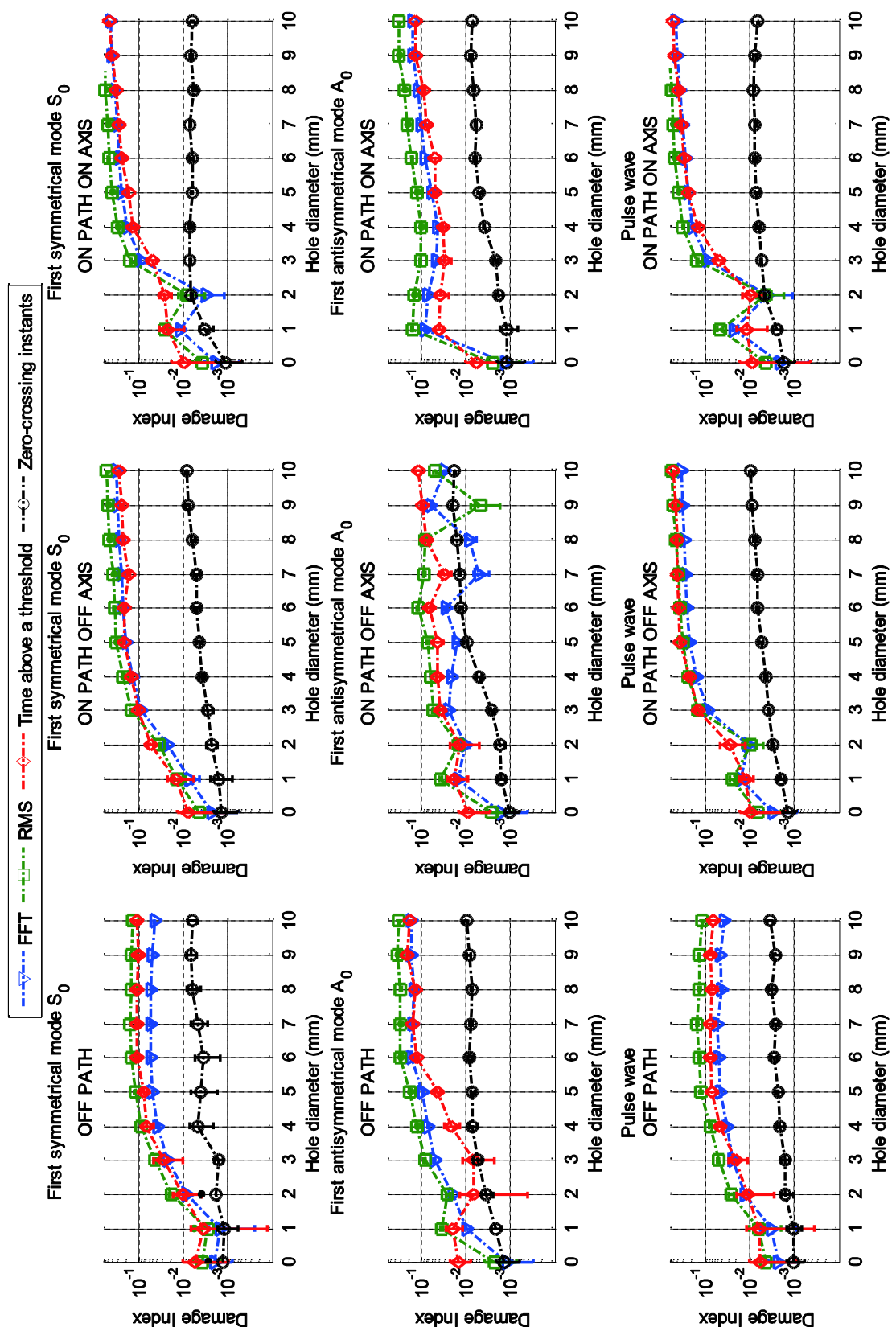

Fig. 4. Evolution of the Damage Indexes as a function of the hole diameter.

self-powered damage detection systems in mind, a particular focus will be placed on the energy requirements for computing the nonlinear DIs.

Figure 5 depicts two simplified schematic blocks for the computation of the nonlinear Damage Indexes. Each of the devices is simply made of a comparator, a counter and a microcontroller. For the first DI based on threshold detection, the principles consist of enabling the counter when the absolute sensed signature is greater than the threshold, hence providing a low-cost way for performing the sum in Eq. (2). After a given time window, the microcontroller samples the output value of the counter, which reflects the time when the absolute signature has been above the threshold. In the case of the second nonlinear approach, the counter keeps running until it is reset by a zero 


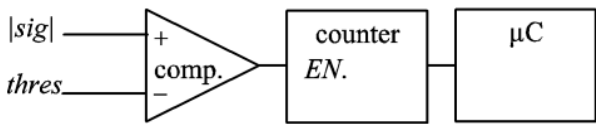

(a)

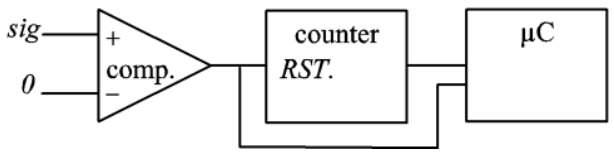

(b)

Fig. 5. Possible implementations (simplified) of the nonlinear Damage Indexes: (a) threshold-based DI; (b) zero crossing-based DI.

crossing detection. Just before the reset, the microcontroller samples the current time period between the two zero crossing instants.

Considering the cost associated with each operation (Table 1), the estimated power consumption of the considered DIs is, considering 1024 samples:

- FFT-based with 200 useful FFT coefficients: $4.1 \mu \mathrm{J}$

- RMS-based: $650 \mathrm{~nJ}$

- Threshold-based: $25 \mathrm{~nJ}$

- Zero-crossing time instant-based with 20 zero-crossing instants: $29 \mathrm{~nJ}$

Hence, this estimation of the required energy for obtaining the DI clearly demonstrates the advantage of the nonlinear approach. While their performance is similar to the FFT-based and RMS-based DI (see Section 4.1), they require almost 200 and 25 times less energy than the FFT-based and RMS-based algorithms, respectively.

Table 1

Energy consumption of typical components for the DI computation

\begin{tabular}{ll}
\hline \multicolumn{1}{c}{ Device } & \multicolumn{1}{c}{ Cost } \\
\hline Microcontroller [11] & $150 \mathrm{pJ}$ per instruction \\
ADC [12] & $30 \mathrm{pJ}$ per 8-bit sample \\
Comparator [13] & $0.02 \mathrm{pJ}$ per comparison \\
Clock (OV-1564-C2, Micro Crystal, Switzerland) & $20 \mathrm{pJ}$ per cycle \\
\hline
\end{tabular}

Although these energy estimations have been obtained using research prototypes, commercially available equivalent components can also be considered, as shown in Table 2. It can be noted that all the devices have similar energy consumption except the ADC and the comparator, which have however similar energy ratio than research devices, hence leading to similar energy gain than previously obtained.

Table 2

Energy consumption of commercially available devices

\begin{tabular}{ll}
\hline \multicolumn{1}{c}{ Device } & \multicolumn{1}{c}{ Cost } \\
\hline Microcontroller PIC NanoWatt 16F688 (Microchip) & $440 \mathrm{pJ}$ per instruction \\
ADC MAX1192 (Maxim) & $5 \mathrm{~nJ}$ per 8-bit sample \\
Comparator TSM9120 (Touchstone semiconductor) & $0.9 \mathrm{pJ}$ per comparison \\
Clock (OV-1564-C2, Micro Crystal, Switzerland) & $20 \mathrm{pJ}$ per cycle \\
\hline
\end{tabular}

Another advantage of the nonlinear DI is that the reference is only a scalar value, which therefore saves storing space and memory reading energy. Finally, it can be noted that the threshold detection permits a self-synchronization of the detection and computation. 


\section{Example of a self-powered damage detection system}

This section proposes a practical implementation of a self-powered SHM scheme based on the Lamb wave interaction with the host structure (Fig. 6). The energy harvesting module uses piezoelectric elements bonded on the host structure which convert ambient vibration into electric energy. In addition, the harvesting system is optimized using the nonlinear Synchronized Switch Harvesting on Inductor approach [14,15]. The system is divided into three parts:

- Autonomous Wireless Transmitter (AWT - [16]): which features an optimized vibration energy harvesting system, a microcontroller and a RF emitter (Fig. 7a). The aim of this module is to generate the Lamb wave.

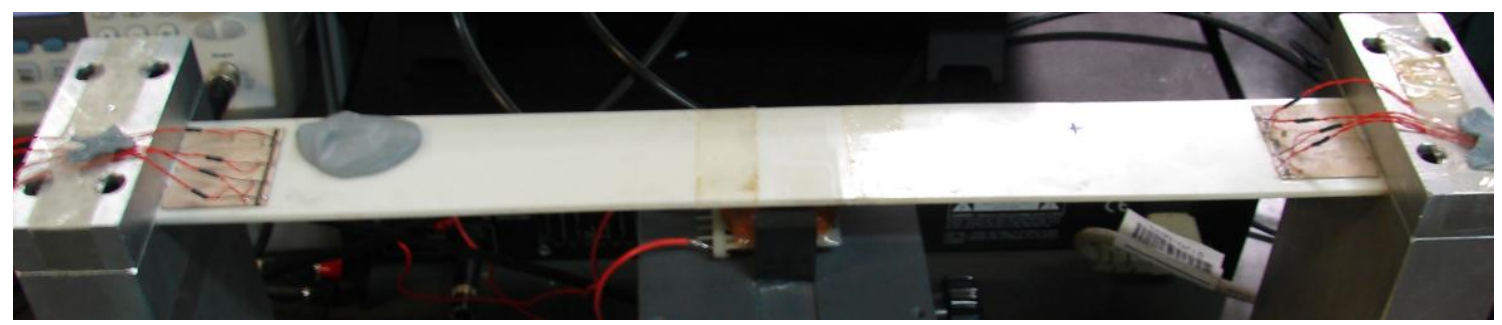

(a)

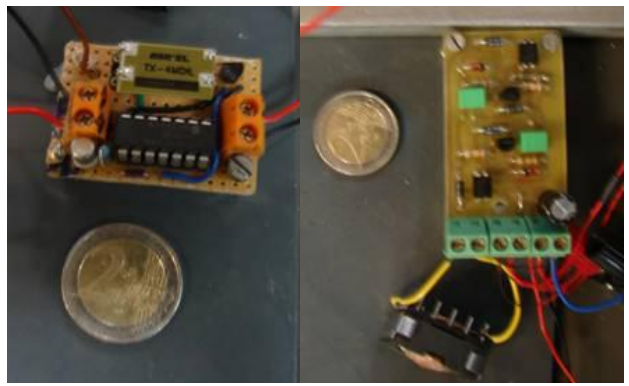

(b)

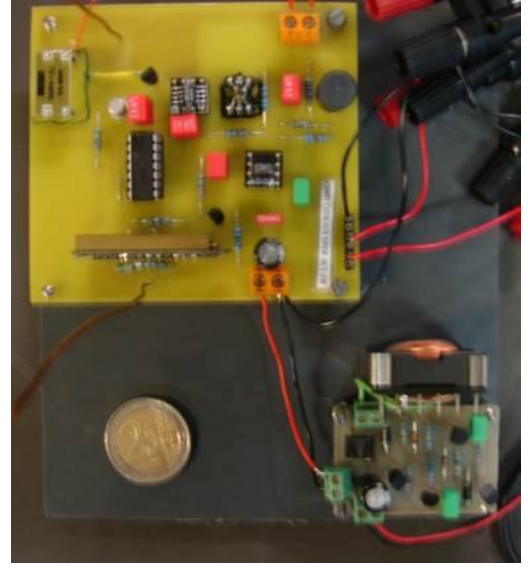

(c)

Fig. 6. Photograph of (a) test structure; (b) AWT module; (c) AWR module.

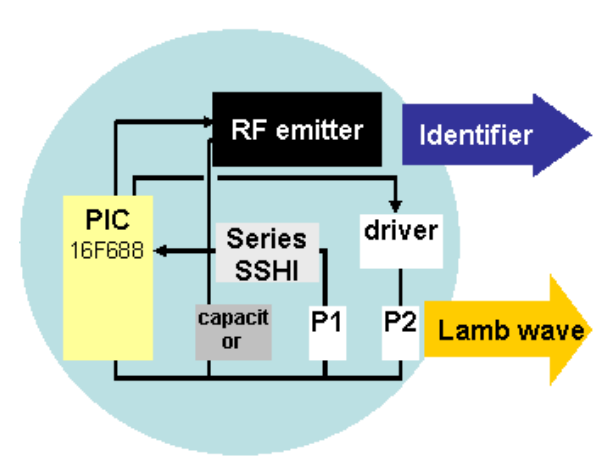

(a)

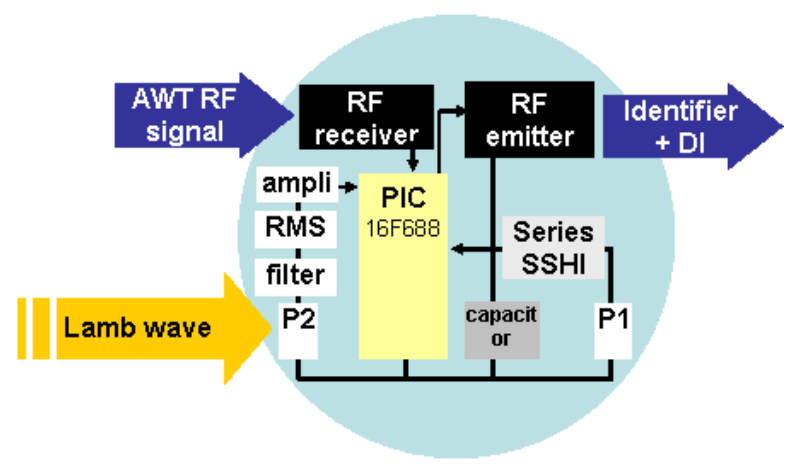

(b)

Fig. 7. Internal structures of (a) Autonomous Wireless Transmitter (AWT); (b) Autonomous Wireless Receiver (AWR). 
- Autonomous Wireless Receiver (AWR - [17]): including the same components than the AWT plus a RF receiver (Fig. 7b). The role of this device is to sense the Lamb wave signature, compute the DI based on RMS value and to send the result to the base station.

- Base Station: externally powered, it consists of gathering all the information within the SHM network and giving a global view of the structural condition.

When designing a self-powered device, the most critical parts are the functions that consume a lot of energy for a given cycle or in other words, the highest product of the power consumption by the active time period, rather than the pure power consumption. Therefore, a careful attention has to be placed both on the components and on the strategy. In order to save as much as energy as possible, the AWR and AWT are in sleep mode during the maximum time. The strategy for the inspection is then defined as follows (Fig. 8):

1. Wake-up of the AWT

2. The AWT sends a RF identifier to the Base Station for time and space identification

3. The AWT sends the Lamb wave by applying a voltage pulse on a piezoelectric insert, and then goes back to sleep mode

4. The AWR intercepts the RF signal send by the AWT, and actives the sensing and computation modules

5. The Lamb wave signature is sensed by the AWR, which computes the Damage Index and sends to the results to the Base Station through RF communication along with its identifier for time and space identification

6. The AWR goes in full sleep mode, including RF reception

7. Just before a new AWT cycle, the AWR wakes up the RF receiver.

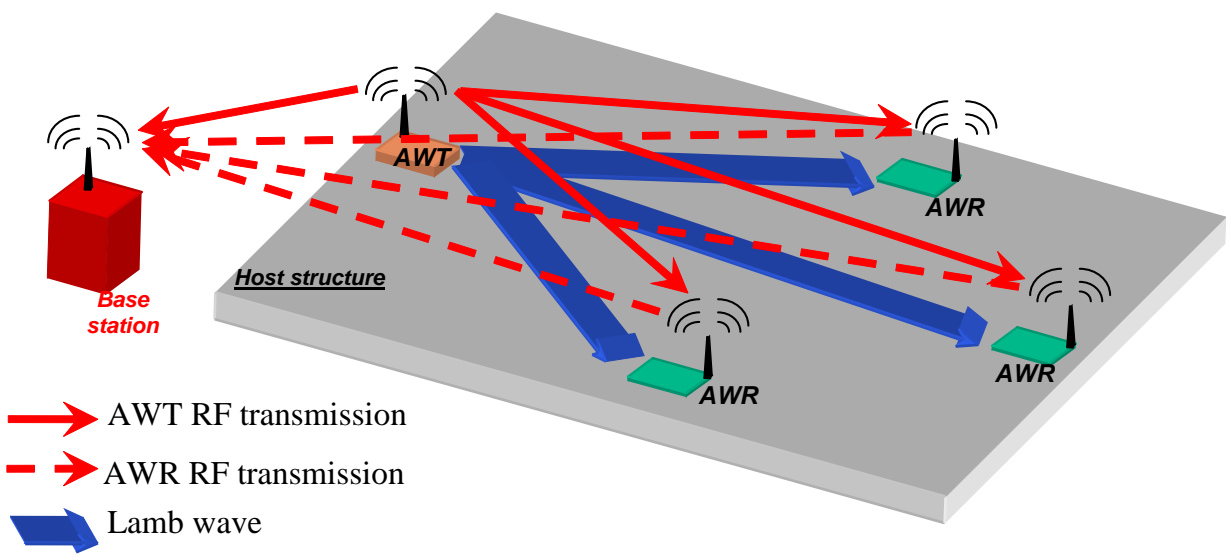

Fig. 8. Wireless communication network.

The associated energies for each operation of the self-powered devices (AWR and AWT) are given in Table 3. It can be noted that the energy for generating the signal also depends on the used transducer. Considering a constant voltage excitation, the energy would increase with the coupling factor and capacitance of the piezoelectric element. Hence, a trade-off exists between the required energy to generate the acoustic wave and the sensibility. For the sensor, no energy is required, as it is piezoelectric and thus active.

Considering the test structure which consists of a clamped-clamped $300 \times 10 \times 0.5 \mathrm{~mm}^{3}$ Bulk Moulding Compound (BMC) beam equipped with four piezoelectric elements on each sides (three for energy harvesting and one for Lamb wave emission/sensing), the minimum stress that allows a positive energy balance for an inspection cycle every 10 seconds is $2 \mathrm{MPa}$, which corresponds to a realistic excitation level. It can be noted that without using the SSHI approach, this minimum stress would be significantly increased, leading to unrealistic condition to make the system working.

The damage identification assessment has been done by simulation of a surface defect obtained through the addition of a small mass of putty on the structure and by 64 inspections for each case. Although such a simulated 
damage may be hard to detect as it is on surface rather than within the volume, results depicted in Fig. 9 demonstrate the ability of the system for identifying the change in the structural condition.

Table 3

Energy consumption for each function considering a single inspection cycle

\begin{tabular}{ll}
\hline \multicolumn{1}{c}{ AWT } & \multicolumn{1}{c}{ AWR } \\
\hline First microcontroller wake-up: $0.8 \mathrm{~mJ}$ & First microcontroller wake-up: $0.8 \mathrm{~mJ}$ \\
RF emission: $0.2 \mathrm{~mJ}$ & RF listening: $0.6 \mathrm{~mJ}$ (listening time: $3 \mathrm{~s}$ ) \\
Lamb wave emission: $0.2 \mathrm{~mJ}$ & DI computation: $0.03 \mathrm{~mJ}$ \\
& RF emission: $0.25 \mathrm{~mJ}$ \\
\hline Total: $1.20 \mathrm{~mJ}$ & Total: $1.68 \mathrm{~mJ}$ \\
\hline
\end{tabular}
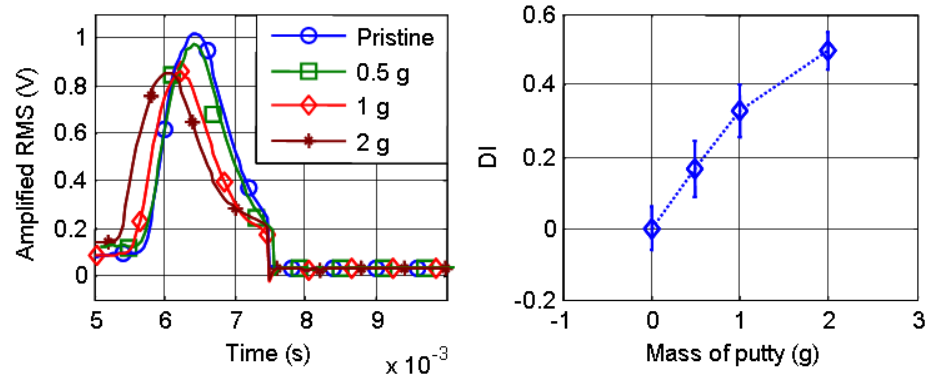

Fig. 9. Obtained results using fake damage (putty).

\section{Conclusion}

This paper investigated low-power SHM techniques intended to be used in a self-powered, autonomous fashion and possibly powered using energy scavenged for the close environment. It has been demonstrated that a nonlinear approach for the computation of a Damage Index permits similar performance compared to classical techniques while significantly decreasing the computation cost and thus the energy requirements, by a factor up to 200 . In addition, power management issues have been discussed, showing the feasibility of a self-powered wireless SHM scheme, therefore allowing an increased safety while reducing the maintenance cost.

\section{References}

[1] S. Roundy, R.K. Wright and J. Rabaey, A study of low level vibrations as a power source for wireless sensor nodes, Computer Communications 26 (2003), 1131-1144.

[2] S.R. Anton and H.A. Sodano, A review of power harvesting using piezoelectric materials (2003-2006), Smart Mater Struct 16 (2007), $\mathrm{R} 1-\mathrm{R} 21$.

[3] C. Boller, Ways and options for aircraft structural health management, Smart Materials and Structures 10 (2001), $432-440$.

[4] E.P. Carden and P. Fanning, Vibration based condition monitoring: A review, Structural Health Monitoring 5(1) (2004), $29-43$.

[5] D. Alleyne and P. Cawley, Optimisation of lamb wave inspection techniques, NDTE International 25 (1992), 11-22.

[6] T. Monnier, Lamb waves-based impact damage monitoring of a stiffened aircraft panel using piezoelectric transducers, Journal of Intelligent Material Systems and Structures 17(5) (2006), 411-421.

[7] T. Monnier, P. Guy, M. Lallart, L. Petit, D. Guyomar and C. Richard, Optimization of signal pre-processing for the integration of cost-effective local intelligence in wireless self-powered Structural Health Monitoring, 3rd International Conference Smart Materials, Structures and Systems (CIMTEC), Acireale, Sicily: Italy, 2008.

[8] M. Frigo and S.G. Johnson, The design and implementation of FFTw3, Proceedings of the IEEE 93(2) (2005), $216-231$.

[9] M. Lallart, T. Monnier and D. Guyomar, Energy-efficient method for embedded in situ structural health monitoring, Structural Health Monitoring 9(1) (2010), 87-98.

[10] D. Guyomar, M. Lallart, K. Li, J.-Y. Gauthier and T. Monnier, A self-synchronizing and low-cost structural health monitoring scheme based on zero crossing detection, Smart Materials and Structures 19 (2010), 045017. 
[11] C. Kok-Leong and G. Bah-Hwee, A low-energy low-voltage asynchronous 8051 microcontroller core, Circuits and Systems, SCAS 2006, Proceedings 2006 IEEE International Symposium, May, Island of Kos, 2006, pp.21-24.

[12] M.D. Scott, B.E. Boser and K.S.J. Pister, An ultralow-energy ADC for smart dust solid-state circuits, Journal of IEEE 38(7) (2003), $1123-1129$.

[13] F. Zhengming and E. Culurciello, An ultra-low power silicon-on-sapphire ADC for energy-scavenging sensors, Circuits and Systems 2006, ISCAS 2006, Proceedings 2006 IEEE International Symposium, 21-24 May, p. 4.

[14] D. Guyomar, A. Badel, E. Lefeuvre and C. Richard, Towards energy harvesting using active materials and conversion improvement by nonlinear processing, IEEE Trans UFFC 52(4) (2005), 584-595.

[15] E. Lefeuvre, A. Badel, C. Richard and D. Guyomar, A comparison between several vibration powered piezoelectric generators for standalone systems, Sens Actuators A 126(2) (2006), 405-416.

[16] D. Guyomar, Y. Jayet, L. Petit, E. Lefeuvre, T. Monnier, C. Richard and M. Lallart, Synchronized switch harvesting applied to self-powered smart systems: Piezoactive microgenerators for autonomous wireless transmitters, Sensors and Actuators A: Physical 138(1) (2007), 151-160.

[17] M. Lallart, D. Guyomar, Y. Jayet, L. Petit, E. Lefeuvre, T. Monnier, P. Guy and C. Richard, Synchronized switch harvesting applied to selfpowered smart systems: Piezoactive microgenerators for autonomous wireless receiver, Sensors and Actuators A: Physical 147(1) (2008), 263-272. 

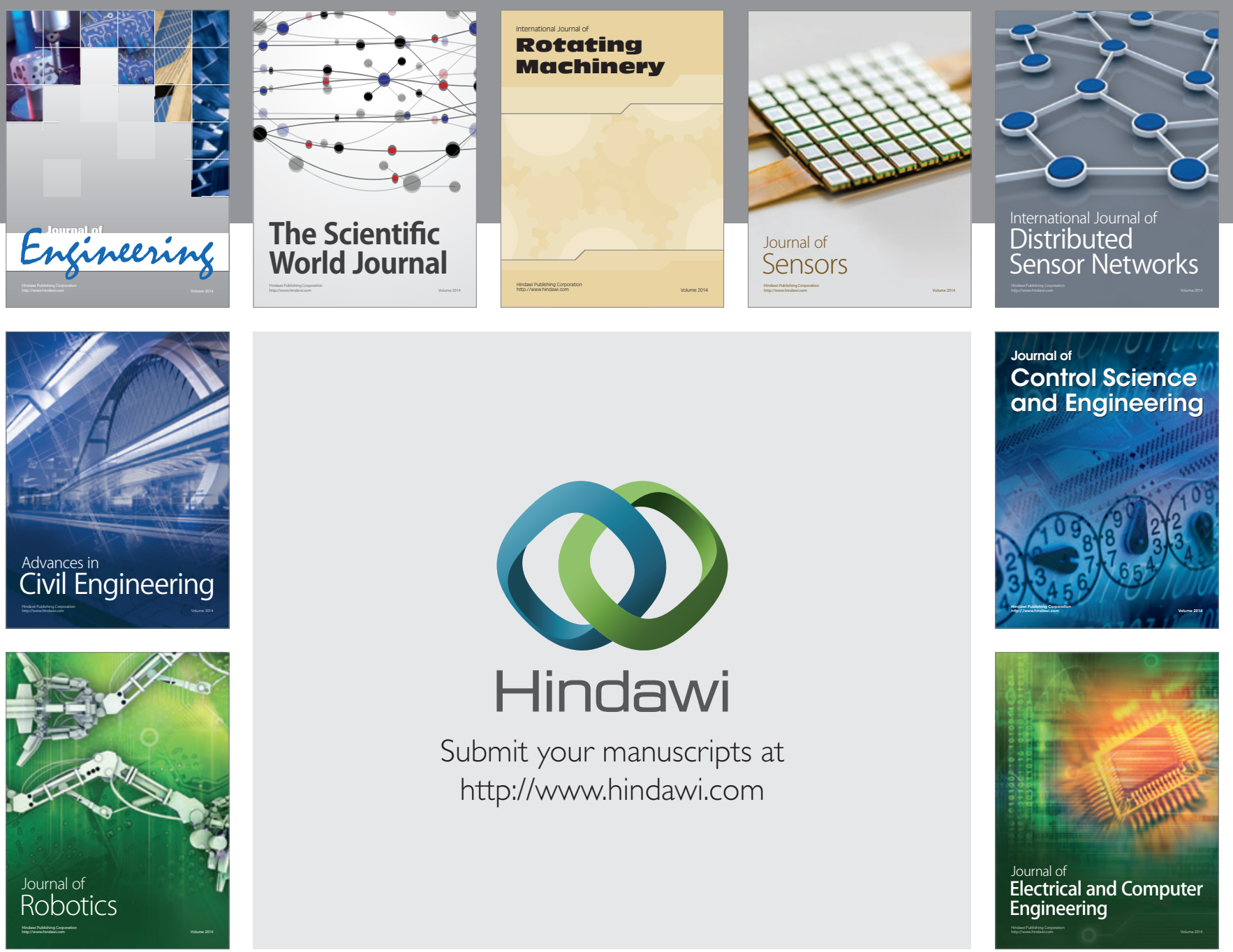

Submit your manuscripts at

http://www.hindawi.com
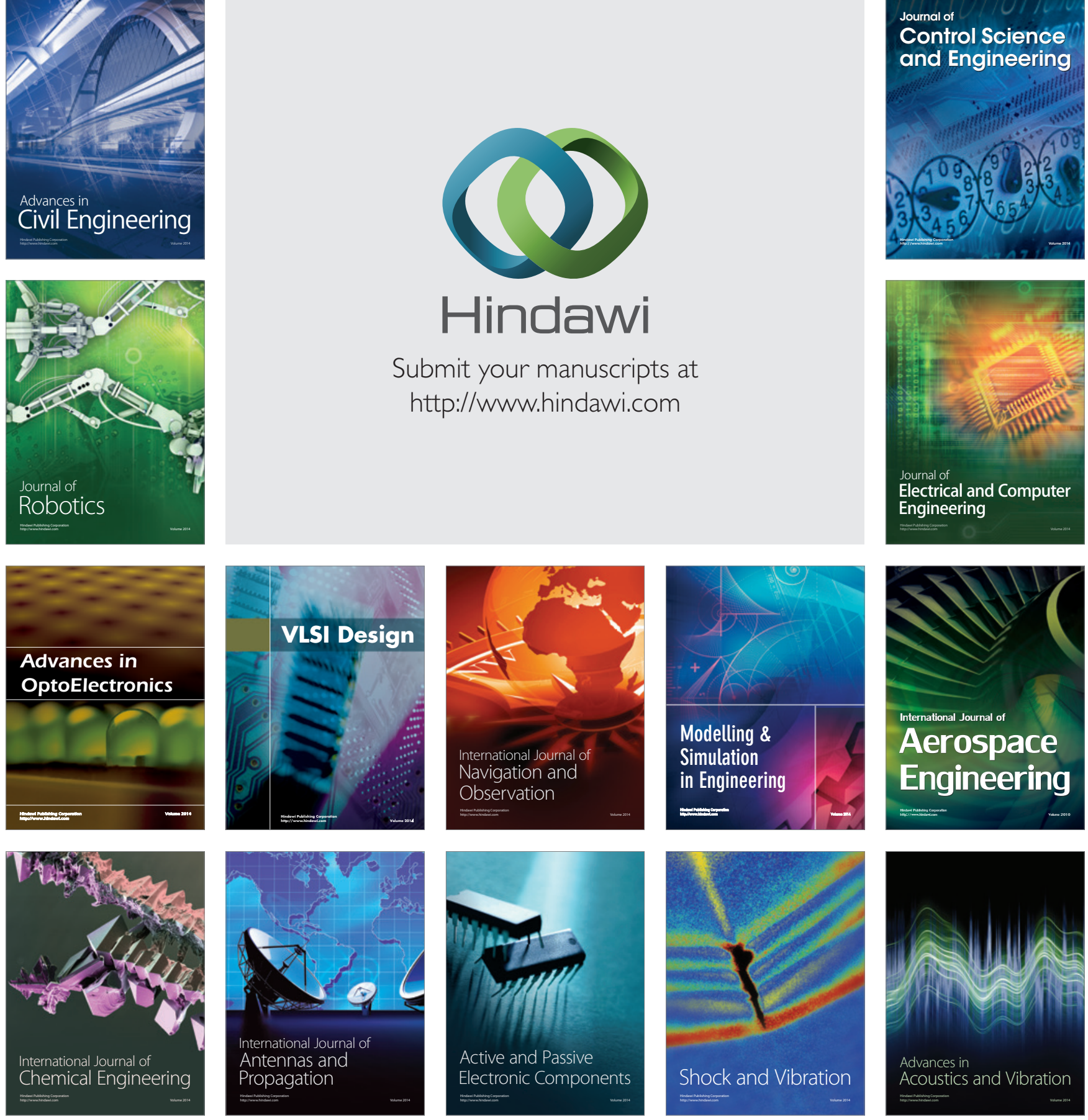